\title{
Drawing Cubic Graphs with at Most Five Slopes
}

\author{
B. Keszegh ${ }^{1}$, J. Pach ${ }^{2,4}$, D. Pálvölgyi ${ }^{3}$, and G. Tóth ${ }^{4}$ \\ ${ }^{1}$ Central European University, Budapest \\ ${ }^{2}$ Courant Institute, NYU, New York \\ ${ }^{3}$ Eötvös University, Budapest \\ 4 A. Rényi Institute of Mathematics, Budapest
}

\begin{abstract}
We show that every graph $G$ with maximum degree three has a straight-line drawing in the plane using edges of at most five different slopes. Moreover, if $G$ is connected and has at least one vertex of degree less than three, then four directions suffice.
\end{abstract}

\section{Introduction}

A planar layout of a graph $G$ is called a straight-line drawing if the vertices of $G$ are represented by distinct points in the plane and every edge is represented by a straight-line segment connecting the corresponding pair of points and not passing through any other point representing a vertex. If it leads to no confusion, in notation and terminology we make no distinction between a vertex and the corresponding point and between an edge and the corresponding segment. The slope of an edge of the layout is the slope of the segment representing it. Layouts with few slopes and few bends have been extensively studied in "graph drawing" [2. In particular, Ungar proved that every three-connected cubic planar graph (i.e., every vertex has degree three) can be drawn using only vertical and horizontal straight-line edges and altogether at most three bends on the outer-face 8 .

Wade and Chu $[9$ introduced the following graph parameter: The slope number of a graph $G$ is the smallest number $s$ with the property that $G$ has a straight-line drawing with edges of at most $s$ distinct slopes and with no bends. Obviously, if $G$ has a vertex of degree $d$, then its slope number is at least $\lceil d / 2\rceil$, because, according to the above definitions, in a proper drawing two edges are not allowed to partially overlap. The question arises whether the slope number can be bounded from above by any function of the maximum degree $d$ (see [3). Barát, Matoušek, and Wood [1] and, independently, Pach and Pálvölgyi [7] proved that the answer is no for $d \geq 5$. Trivially, every graph of maximum degree two has slope number at most three. What happens if $d=3$ or 4 ?

The aim of this note is to establish the following theorem.

Theorem 1. Every graph of maximum degree at most three has slope number at most five.

Our terminology is somewhat unorthodox: by the slope of a line $\ell$, we mean the angle $\alpha$ modulo $\pi$ such that a counterclockwise rotation through $\alpha$ takes the 
$x$-axis to a position parallel to $\ell$. The slope of an edge (segment) is the slope of the line containing it. In particular, the slopes of the lines $y=x$ and $y=-x$ are $\pi / 4$ and $-\pi / 4$, and they are called Northeast (or Southwest) and Northwest (or Southeast) lines, respectively.

For any two points $p_{1}=\left(x_{1}, y_{1}\right), p_{2}=\left(x_{2}, y_{2}\right) \in \mathbf{R}^{2}$, we say that $p_{2}$ is to the North (or to the South of $p_{1}$ if $x_{2}=x_{1}$ and $y_{2}>y_{1}$ (or $y_{2}<y_{1}$ ). Analogously, we say that $p_{2}$ is to the Northeast (to the Northwest) of $p_{1}$ if $y_{2}>y_{1}$ and $p_{1} p_{2}$ is a Northeast (Northwest) line. Directions are often abbreviated by their first letters: N, NE, E, SE, etc. These four directions are referred to as basic. That is, a line $\ell$ is said to be of one of the four basic directions if $\ell$ is parallel to one of the axes or to one of the NE and NW lines $y=x$ and $y=-x$.

The main tool of our proof is the following result of independent interest.

Theorem 2. Let $G$ be a connected graph that is not a cycle and whose every vertex has degree at most three. Suppose that $G$ has at least one vertex of degree less than three, and denote by $v_{1}, \ldots, v_{m}$ the vertices of degree at most two $(m \geq 1)$.

Then, for any sequence $x_{1}, x_{2}, \ldots, x_{m}$ of real numbers, linearly independent over the rationals, $G$ has a straight-line drawing with the following properties:

(1) Vertex $v_{i}$ is mapped into a point with $x$-coordinate $x\left(v_{i}\right)=x_{i}(1 \leq i \leq m)$;

(2) The slope of every edge is $0, \pi / 2, \pi / 4$, or $-\pi / 4$.

(3) No vertex is to the North of any vertex of degree two.

(4) No vertex is to the North or to the Northwest of any vertex of degree one.

It was shown by Dujmović at al. 3. that every planar graph with maximum degree three has a drawing with noncrossing straight-line edges of at most three different slopes, except that three edges of the outer-face may have a bend.

Eppstein [6], Duncan et al. 4], and Barát et al. [1] studied another parameter, the geometric thickness of a graph, which is closely related to the slope number.

Max Engelstein [5], a student from Stuyvesant High School, New York has shown that every graph of maximum degree three that has a Hamiltonian cycle can be drawn with edges of at most five different slopes.

\section{Embedding Cycles}

Let $C$ be a straight-line drawing of a cycle in the plane. A vertex $v$ of $C$ is said to be a turning point if the slopes of the two edges meeting at $v$ are not the same.

We start with two simple auxiliary statements.

Lemma 2.1. Let $C$ be a straight-line drawing of a cycle such that the slope of every edge is $0, \pi / 4$, or $-\pi / 4$. Then the $x$-coordinates of the vertices of $C$ are not independent over the rational numbers.

Moreover, there is a vanishing linear combination of the $x$-coordinates of the vertices, with as many nonzero (rational) coefficients as many turning points Chas. 
Proof. Let $v_{1}, v_{2}, \ldots, v_{n}$ denote the vertices of $C$ in cyclic order $\left(v_{n+1}=v_{1}\right)$. Let $x\left(v_{i}\right)$ and $y\left(v_{i}\right)$ be the coordinates of $v_{i}$. For any $i(1 \leq i \leq n)$, we have $y\left(v_{i+1}\right)-y\left(v_{i}\right)=\lambda_{i}\left(x\left(v_{i+1}\right)-x\left(v_{i}\right)\right)$, where $\lambda_{i}=0,1$, or -1 , depending on the slope of the edge $v_{i} v_{i+1}$. Adding up these equations for all $i$, the left-hand sides add up to zero, while the sum of the right-hand sides is a linear combination of the numbers $x\left(v_{1}\right), x\left(v_{2}\right), \ldots, x\left(v_{n}\right)$ with integer coefficients of absolute value at most two.

Thus, we are done with the first statement of the lemma, unless all of these coefficients are zero. Obviously, this could happen if and only if $\lambda_{1}=\lambda_{2}=$ $\ldots=\lambda_{n}$, which is impossible, because then all points of $C$ would be collinear, contradicting our assumption that in a proper straight-line drawing no edge is allowed to pass through any vertex other than its endpoints.

To prove the second statement, it is sufficient to notice that the coefficient of $x\left(v_{i}\right)$ vanishes if and only if $v_{i}$ is not a turning point.

Lemma 2.1 shows that Theorem 2 does not hold if $G$ is a cycle. Nevertheless, according to the next claim, cycles satisfy a very similar condition. Observe, that the main difference is that here we have an exceptional vertex, denoted by $v_{0}$.

Lemma 2.2. Let $C$ be a cycle with vertices $v_{0}, v_{1}, \ldots, v_{m}$, in this cyclic order.

Then, for any real numbers $x_{1}, x_{2}, \ldots, x_{m}$, linearly independent over the rationals, $C$ has a straight-line drawing with the following properties:

(1) Vertex $v_{i}$ is mapped into a point with $x$-coordinate $x\left(v_{i}\right)=x_{i}(1 \leq i \leq m)$;

(2) The slope of every edge is $0, \pi / 4$, or $-\pi / 4$.

(3) No vertex is to the North of any other vertex.

(4) No vertex has a larger $y$-coordinate than $y\left(v_{0}\right)$.

Proof. We can assume without loss of generality that $x_{2}>x_{1}$. Place $v_{1}$ at any point $\left(x_{1}, 0\right)$ of the $x$-axis. Assume that for some $i<m$, we have already determined the positions of $v_{1}, v_{2}, \ldots v_{i}$, satisfying conditions (1)-(3). If $x_{i+1}>$ $x_{i}$, then place $v_{i+1}$ at the (unique) point Southeast of $v_{i}$, whose $x$-coordinate is $x_{i+1}$. If $x_{i+1}<x_{i}$, then put $v_{i+1}$ at the point West of $x_{i}$, whose $x$-coordinate is $x_{i+1}$. Clearly, this placement of $v_{i+1}$ satisfies (1)-(3), and the segment $v_{i} v_{i+1}$ does not pass through any point $v_{j}$ with $j<i$.

After $m$ steps, we obtain a noncrossing straight-line drawing of the path $v_{1} v_{2} \ldots v_{m}$, satisfying conditions (1)-(3). We still have to find a right location for $v_{0}$. Let $R_{W}$ and $R_{S E}$ denote the rays (half-lines) starting at $v_{1}$ and pointing to the West and to the Southeast. Further, let $R$ be the ray starting at $v_{m}$ and pointing to the Northeast. It follows from the construction that all points $v_{2}, \ldots, v_{m}$ lie in the convex cone below the $x$-axis, enclosed by the rays $R_{W}$ and $R_{S E}$.

Place $v_{0}$ at the intersection point of $R$ and the $x$-axis. Obviously, the segment $v_{m} v_{0}$ does not pass through any other vertex $v_{j}(0<j<m)$. Otherwise, we could find a drawing of the cycle $v_{j} v_{j+1} \ldots v_{m}$ with slopes $0, \pi / 4$, and $-\pi / 4$. By Lemma 2.1, this would imply that the numbers $x_{j}, x_{j+1}, \ldots, x_{m}$ are not independent over the rationals, contradicting our assumption. It is also clear that the horizontal segment $v_{0} v_{1}$ does not pass through any vertex different from its 
endpoints because all other vertices are below the horizontal line determined by $v_{0} v_{1}$. Hence, we obtain a proper straight-line drawing of $C$ satisfying conditions (1), (2), and (4).

It remains to verify $(3)$. The only thing we have to check is that $x\left(v_{0}\right)$ does not coincide with any other $x\left(v_{i}\right)$. Suppose it does, that is, $x\left(v_{0}\right)=x\left(v_{i}\right)=x_{i}$ for some $i>0$. By the second statement of Lemma 2.1, there is a vanishing linear combination

$$
\lambda_{0} x\left(v_{0}\right)+\lambda_{1} x_{1}+\lambda_{2} x_{2}+\ldots+\lambda_{m} x_{m}=0
$$

with rational coefficients $\lambda_{i}$, where the number of nonzero coefficients is at least the number of turning points, which cannot be smaller than three. Therefore, if in this linear combination we replace $x\left(v_{0}\right)$ by $x_{i}$, we still obtain a nontrivial rational combination of the numbers $x_{1}, x_{2}, \ldots, x_{m}$. This contradicts our assumption that these numbers are independent over the rationals.

\section{The Embedding Procedure: Proof of Theorem 2}

First we settle Theorem 2 in a special case.

Lemma 3.1 Let $m, k \geq 2$ and let $G$ be a graph consisting of two disjoint cycles, $C=\left\{v_{0}, v_{1}, \ldots, v_{m}\right\}$ and $C^{\prime}=\left\{v_{0}^{\prime}, v_{1}^{\prime}, \ldots, v_{m}^{\prime}\right\}$, connected by a single edge $v_{0} v_{0}^{\prime}$.

Then, for any sequence $x_{1}, x_{2}, \ldots, x_{m}, x_{1}^{\prime}, x_{2}^{\prime}, \ldots, x_{k}^{\prime}$ of real numbers, linearly independent over the rationals, $G$ has a straight-line drawing satisfying the following conditions:

(1) The vertices $v_{i}$ and $v_{j}^{\prime}$ are mapped into points with $x$-coordinates $x\left(v_{i}\right)=$ $x_{i}(1 \leq i \leq m)$ and $x\left(v_{j}\right)=x_{j}^{\prime}(1 \leq j \leq k)$.

(2) The slope of every edge is $0, \pi / 2, \pi / 4$, or $-\pi / 4$.

(3) No vertex is to the North of any vertex of degree two.

Proof of Lemma 3.1. Apply Lemma 2.2 to cycle $C$ with vertices $v_{0}, v_{1}, \ldots, v_{m}$, with assigned $x$-coordinates $x_{1}, x_{2}, \ldots, x_{m}$, and analogously, to the cycle $C^{\prime}$, with vertices $v_{0}^{\prime}, v_{1}^{\prime}, \ldots, v_{k}^{\prime}$ and assigned $x$-coordinates $x_{1}^{\prime}, x_{2}^{\prime}, \ldots, x_{k}^{\prime}$. For simplicity, the resulting drawings are also denoted by $C$ and $C^{\prime}$.

Let $x_{0}$ and $x_{0}^{\prime}$ denote the $x$-coordinates of $v_{0} \in C$ and $v_{0}^{\prime} \in C^{\prime}$. It follows from Lemma 2.1 that $x_{0}$ is a linear combination of $x_{1}, x_{2}, \ldots, x_{m}$, and $x_{0}^{\prime}$ is a linear combination of $x_{1}^{\prime}, x_{2}^{\prime}, \ldots, x_{k}^{\prime}$ ) with rational coefficients. Therefore, if $x_{0}=x_{0}^{\prime}$, then there is a nontrivial linear combination of $x_{1}, x_{2}, \ldots, x_{m}, x_{1}^{\prime}, x_{2}^{\prime}, \ldots, x_{k}^{\prime}$ that gives 0 , contradicting the assumption that these numbers are independent over the rationals. Thus, we can conclude that $x_{0} \neq x_{0}^{\prime}$. Assume without loss of generality that $x_{0}<x_{0}^{\prime}$. Reflect $C^{\prime}$ about the $x$-axis, and shift it in the vertical direction so that $v_{0}^{\prime}$ ends up to the Northeast from $v_{0}$. Clearly, we can add the missing edge $v_{0} v_{0}^{\prime}$. Let $D$ denote the resulting drawing of $G$. We claim that $D$ meets all the requirements of the Theorem. Conditions (1), (2), and (3) are obviously satisfied, we only have to check that no vertex lies in the interior of an edge. It follows from Lemma 2.2 that the $y$-coordinates of $v_{1}, \ldots, v_{m}$ are all smaller than or equal to the $y$-coordinate of $v_{0}$ and the $y$-coordinates of 
$v_{1}^{\prime}, \ldots, v_{k}^{\prime}$ are all greater than or equal to the $y$-coordinate of $v_{0}^{\prime}$. We also have $y\left(v_{0}\right)<y\left(v_{0}^{\prime}\right)$. Therefore, there is no vertex in the interior of $v_{0} v_{0}^{\prime}$. Moreover, no edge of $C$ (resp. $C^{\prime}$ ) can contain any vertex of $v_{0}^{\prime}, v_{1}^{\prime}, \ldots, v_{k}^{\prime}\left(\right.$ resp. $\left.v_{0}, v_{1}, \ldots, v_{m}\right)$ in its interior.

The rest of the proof is by induction on the number of vertices of $G$. The statement is trivial if the number of vertices is at most two. Suppose that we have already established Theorem 2 for all graphs with fewer than $n$ vertices.

Suppose that $G$ has $n$ vertices, it is not a cycle and not the union of two cycles connected by one edge. Let $v_{1}, v_{2}, \ldots, v_{m}$ be the vertices of $G$ with degree less than three, and let the $x$-coordinates assigned to them be $x_{1}, x_{2}, \ldots, x_{m}$.

We distinguish several cases.

\section{Case 1: $G$ has a vertex of degree one.}

Assume, without loss of generality, that $v_{1}$ is such a vertex. If $G$ has no vertex of degree three, then it consists of a simple path $P=v_{1} v_{2} \ldots v_{m}$, say. Place $v_{m}$ at the point $\left(x_{m}, 0\right)$. In general, assuming that $v_{i+1}$ has already been embedded for some $i<m$, and $x_{i}<x_{i+1}$, place $v_{i}$ at the point West of $v_{i+1}$, whose $x$ coordinate is $x_{i}$. If $x_{i}>x_{i+1}$, then put $v_{i}$ at the point Northeast of $v_{i+1}$, whose $x$-coordinate is $x_{i}$. The resulting drawing of $G=P$ meets all the requirements of the theorem. To see this, it is sufficient to notice that if $v_{j}$ would be Northwest of $v_{m}$ for some $j<m$, then we could apply Lemma 2.1 to the cycle $v_{j} v_{j+1} \ldots v_{m}$, and conclude that the numbers $x_{j}, x_{j+1}, \ldots, x_{m}$ are dependent over the rationals. This contradicts our assumption.

Assume next that $v_{1}$ is of degree one, and that $G$ has at least one vertex of degree three. Suppose without loss of generality that $v_{1} v_{2} \ldots v_{k} w$ is a path in $G$, whose internal vertices are of degree two, but the degree of $w$ is three. Let $G^{\prime}$ denote the graph obtained from $G$ by removing the vertices $v_{1}, v_{2}, \ldots, v_{k}$. Obviously, $G^{\prime}$ is a connected graph, in which the degree of $w$ is two.

If $G^{\prime}$ is a cycle, then apply Lemma 2.2 to $C=G^{\prime}$ with $w$ playing the role of the vertex $v_{0}$ which has no preassigned $x$-coordinate. We obtain an embedding of $G^{\prime}$ with edges of slopes $0, \pi / 4$, and $-\pi / 4$ such that $x\left(v_{i}\right)=x_{i}$ for all $i>k$ and there is no vertex to the North, to the Northeast, or to the Northwest of $w$. By Lemma 2.1, the numbers $x(w), x_{k+1}, \ldots, x_{m}$ are not independent over the rationals. Therefore, $x(w) \neq x_{k}$, so we can place $v_{k}$ at the point to the Northwest or to the Northeast of $w$, whose $x$-coordinate is $x_{k}$, depending on whether $x(w)>x_{k}$ or $x(w)<x_{k}$. After this, embed $v_{k-1}, \ldots, v_{1}$, in this order, so that $v_{i}$ is either to the Northeast or to the West of $v_{i+1}$ and $x\left(v_{i}\right)=x_{i}$. According to property (4) in Lemma 2.1, the path $v_{1} v_{2} \ldots v_{k}$ lies entirely above $G^{\prime}$, so that no point of $G$ can lie to the North or to the Northwest of $v_{1}$.

If $G^{\prime}$ is not a cycle, then use the induction hypothesis to find an embedding of $G^{\prime}$ that satisfies all conditions of Theorem 2, with $x(w)=x_{k}$ and $x\left(v_{i}\right)=x_{i}$ for every $i>k$. Now place $v_{k}$ very far from $w$, to the North of it, and draw $v_{k-1}, \ldots, v_{1}$, in this order, in precisely the same way as in the previous case. Now if $v_{k}$ is far enough, then none of the points $v_{k}, v_{k-1}, \ldots, v_{1}$ is to the Northwest or to the Northeast of any vertex of $G^{\prime}$. It remains to check that condition (4) 
is true for $v_{1}$, but this follows from the fact that there is no point of $G$ whose $y$-coordinate is larger than that of $v_{1}$.

From now on, we can and will assume that $G$ has no vertex of degree one.

A graph with four vertices and five edges between them is said to be a $\Theta$-graph.

Case 2: $G$ contains a $\Theta$-subgraph.

Suppose that $G$ has a $\Theta$-subgraph with vertices $a, b, c, d$, and edges $a b, b c, a c, a d$, $b d$. If neither $c$ nor $d$ has a third neighbor, then $G$ is identical to this graph, which can easily be drawn in the plane with all conditions of the theorem satisfied.

If $c$ and $d$ are connected by an edge, then all four points of the $\Theta$-subgraph have degree three, so that $G$ has no other vertices. So $G$ is a complete graph of four vertices, and it has a drawing that meets the requirements.

Suppose that $c$ and $d$ have a common neighbor $e \neq a, b$. If $e$ has no further neighbor, then $a, b, c, d, e$ are the only vertices of $G$, and again we can easily find a proper drawing. Thus, we can assume that $e$ has a third neighbor $f$. By the induction hypothesis, $G^{\prime}=G \backslash\{a, b, c, d, e\}$ has a drawing satisfying the conditions of Theorem 2. In particular, no vertex of $G^{\prime}$ is to the North of $f$ (and to the Northwest of $f$, provided that the degree of $f$ in $G^{\prime}$ is one). Further, consider a drawing $H$ of the subgraph of $G$ induced by the vertices $a, b, c, d, e$, which satisfies the requirements. We distinguish two subcases.

If the degree of $f$ in $G^{\prime}$ is one, then take a very small homothetic copy of $H$ (i.e., similar copy in parallel position), and rotate it about $e$ in the clockwise direction through $3 \pi / 4$. There is no point of this drawing, denoted by $H^{\prime}$, to the Southeast of $e$, so that we can translate it into a position in which $e$ is to the Northwest of $f \in V\left(G^{\prime}\right)$ and very close to it. Connecting now $e$ to $f$, we obtain a drawing of $G$ satisfying the conditions. Note that it was important to make $H^{\prime}$ very small and to place it very close to $f$, to make sure that none of its vertices is to the North of any vertex of $G^{\prime}$ whose degree is at most two, or to the Northwest of any vertex of degree one (other than $f$ ).

If the degree of $f$ in $G^{\prime}$ is two, then we follow the same procedure, except that now $H^{\prime}$ is a small copy of $H$, rotated by $\pi$. We translate $H^{\prime}$ into a position in which $e$ is to the North of $f$, and connect $e$ to $f$ by a vertical segment. It is again clear that the resulting drawing of $G$ meets the requirements in Theorem 2. Thus, we are done if $c$ and $d$ have a common neighbor $e$.

Suppose now that only one of $c$ and $d$ has a third neighbor, different from $a$ and $b$. Suppose, without loss of generality, that this vertex is $c$, so that the degree of $d$ is two. Then in $G^{\prime}=G \backslash\{a, b, d\}$, the degree of $c$ is one. Apply the induction hypothesis to $G^{\prime}$ so that the $x$-coordinate originally assigned to $d$ is now assigned to $c$ (which had no preassigned $x$-coordinate in $G$ ). In the resulting drawing, we can easily reinsert the remaining vertices, $a, b, d$, by adding a very small square whose lowest vertex is at $c$ and whose diagonals are parallel to the coordinate axes. The highest vertex of this square will represent $d$, and the other two vertices will represent $a$ and $b$.

We are left with the case when both $c$ and $d$ have a third neighbor, other than $a$ and $b$, but these neighbors are different. Denote them by $c^{\prime}$ and $d^{\prime}$, respectively. Create a new graph $G^{\prime}$ from $G$, by removing $a, b, c, d$ and adding a new vertex 
$v$, which is connected to $c^{\prime}$ and $d^{\prime}$. Draw $G^{\prime}$ using the induction hypothesis, and reinsert $a, b, c, d$ in a small neighborhood of $v$ so that they form the vertex set of a very small square with diagonal $a b$. (See Figure 1.) As before, we have to choose this square sufficiently small to make sure that $a, b, c, d$ are not to the North of any vertex $w \neq c^{\prime}, d^{\prime}, v$ of $G^{\prime}$, whose degree is at most two, or to the Northwest of any vertex of degree one. Thus, we are done if $G$ has a $\Theta$-subgraph.

So, from now on we assume that $G$ has no $\Theta$-subgraph.
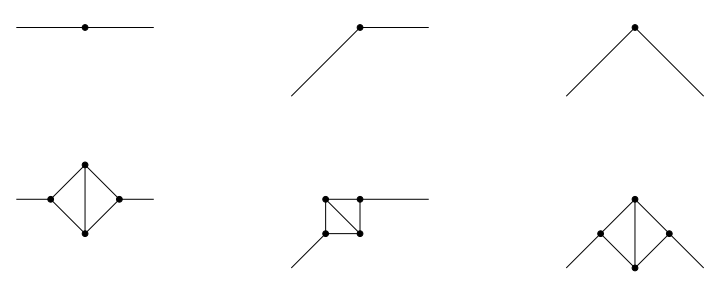

Fig. 1. Replacing $v$ by $\Theta$

Case 3: $G$ has no cycle that passes through a vertex of degree two.

Since $G$ is not three-regular, it contains at least one vertex of degree two. Consider a decomposition of $G$ into two-connected blocks and edges. If a block contains a vertex of degree two, then it consists of a single edge. The block decomposition has a treelike structure, so that there is a vertex $w$ of degree two, such that $G$ can be obtained as the union of two graphs, $G_{1}$ and $G_{2}$, having only the vertex $w$ in common, and there is no vertex of degree two in $G_{1}$.

By the induction hypothesis, for any assignment of rationally independent $x$-coordinates to all vertices of degree less than three, $G_{1}$ and $G_{2}$ have proper straight-line embeddings (drawings) satisfying conditions (1)-(4) of the theorem. The only vertex of $G_{1}$ with a preassigned $x$-coordinate is $w$. Applying a vertical translation, if necessary, we can achieve that in both drawings $w$ is mapped into the same point. Using the induction hypothesis, we obtain that in the union of these two drawings, there is no vertex in $G_{1}$ or $G_{2}$ to the North or to the Northwest of $w$, because the degree of $w$ in $G_{1}$ and $G_{2}$ is one (property (4)). This is stronger than what we need: indeed, in $G$ the degree of $w$ is two, so that we require only that there is no point of $G$ to the North of $w$ (property (3)).

The superposition of the drawings of $G_{1}$ and $G_{2}$ satisfies all conditions of the theorem. Only two problems may occur:

1. A vertex of $G_{1}$ may end up at a point to the North of a vertex of $G_{2}$ with degree two.

2. The (unique) edges in $G_{1}$ and $G_{2}$, incident to $w$, may partially overlap.

Notice that both of these events can be avoided by enlarging the drawing of $G_{1}$, if necessary, from the point $w$, and rotating it about $w$ by $\pi / 4$ in the clockwise direction. The latter operation is needed only if problem 2 occurs. This completes 
the induction step in the case when $G$ has no cycle passing through a vertex of degree two.

It remains to analyze the last case.

Case 4: $G$ has a cycle passing through a vertex of degree two.

By assumption, $G$ itself is not a cycle. Therefore, we can also find a shortest cycle $C$ whose vertices are denoted by $v, u_{1}, \ldots, u_{k}$, in this order, where the degree of $v$ is two and the degree of $u_{1}$ is three. The length of $C$ is $k+1$.

It follows from the minimality of $C$ that $u_{i}$ and $u_{j}$ are not connected by an edge of $G$, for any $|i-j|>1$. Moreover, if $|i-j|>2$, then $u_{i}$ and $u_{j}$ do not even have a common neighbor $(1 \leq i \neq j \leq k)$. This implies that any vertex $v \in V(G \backslash C)$ has at most three neighbors on $C$, and these neighbors must be consecutive on $C$. However, three consecutive vertices of $C$, together with their common neighbor, would form a $\Theta$-subgraph in $G$ (see Case 2). Hence, we can assume that every vertex belonging to $G \backslash C$ is joined to at most two vertices on C.

Let $B_{i}$ denote the set of all vertices of $G \backslash C$ that have precisely $i$ neighbors on $C(i=0,1,2)$. Thus, we have $V(G \backslash C)=B_{0} \cup B_{1} \cup B_{2}$. Further, $B_{1}=B_{1}^{2} \cup B_{1}^{3}$, where an element of $B_{1}$ belongs to $B_{1}^{2}$ or $B_{1}^{3}$, according to whether its degree in $G$ is two or three.

Consider the list $v_{1}, v_{2}, \ldots, v_{m}$ of all vertices of $G$ with degree two. (Recall that we have already settled the case when $G$ has a vertex of degree one.) Assume without loss of generality that $v_{1}=v$ and that $v_{i}$ belongs to $C$ if and only if $1 \leq i \leq j$ for some $j \leq m$.

Let $\mathbf{x}$ denote the assignment of $x$-coordinates to the vertices of $G$ with degree two, that is, $\mathbf{x}=\left(x\left(v_{1}\right), x\left(v_{2}\right), \ldots, x\left(v_{m}\right)\right)=\left(x_{1}, x_{2}, \ldots, x_{m}\right)$. Given $G$, $C, \mathbf{x}$, and a real parameter $L$, we define the following so-called EMBEDDing Procedure $(G, C, \mathbf{x}, L)$ to construct a drawing of $G$ that meets all requirements of the theorem, and satisfies the additional condition that the $y$-coordinate of every vertex of $C$ is at least $L$ higher than the $y$-coordinates of all other vertices of $G$.

STEP 1: If $G^{\prime}:=G \backslash C$ is not a cycle, then construct recursively a drawing of $G^{\prime}:=G \backslash C$ satisfying the conditions of Theorem 2 with the assignment $\mathbf{x}^{\prime}$ of $x$-coordinates $x\left(v_{i}\right)=x_{i}$ for $j<i \leq m$, and $x\left(u_{1}^{\prime}\right)=x_{1}$, where $u_{1}^{\prime}$ is the unique vertex in $G \backslash C$, connected by an edge to $u_{1} \in V(C)$.

If $G^{\prime}=G \backslash C$ is a cycle, then, by assumption, there are at least two edges between $C$ and $G^{\prime}$. One of them connects $u_{1}$ to $u_{1}^{\prime}$. Let $u_{\alpha} u_{\alpha}^{\prime}$ be another such edge, where $u_{\alpha} \in C$ and $u_{\alpha}^{\prime} \in G^{\prime}$. Since the maximum degree is three, $u_{1}^{\prime} \neq u_{\alpha}^{\prime}$. Now construct recursively a drawing of $G^{\prime}:=G \backslash C$ satisfying the conditions of Lemma 2.2, with the assignment $\mathbf{x}^{\prime}$ of $x$-coordinates $x\left(v_{i}\right)=x_{i}$ for $j<i \leq m$, $x\left(u_{1}^{\prime}\right)=x_{1}$, and with exceptional vertex $u_{\alpha}^{\prime}$.

STEP 2: For each element of $B_{1}^{2} \cup B_{2}$, take two rays starting at this vertex, pointing to the Northwest and to the North. Further, take a vertical ray pointing to the North from each element of $B_{1}^{3}$ and each element of the set $B_{\mathbf{x}}:=$ 
$\left\{\left(x_{2}, 0\right),\left(x_{3}, 0\right), \ldots,\left(x_{j}, 0\right)\right\}$. Let $\mathcal{R}$ denote the set of all of these rays. Choose the $x$-axis above all points of $G^{\prime}$ and all intersection points between the rays in $\mathcal{R}$.

For any $u_{h}(1 \leq h \leq k)$ whose degree in $G$ is three, define $N\left(u_{h}\right)$ as the unique neighbor of $u_{h}$ in $G \backslash C$. If $u_{h}$ has degree two in $G$, then $u_{h}=v_{i}$ for some $1 \leq i \leq j$, and let $N\left(u_{h}\right)$ be the point $\left(x_{i}, 0\right)$.

STEP 3: Recursively place $u_{1}, u_{2}, \ldots u_{k}$ on the rays belonging to $\mathcal{R}$, as follows. Place $u_{1}$ on the vertical ray starting at $N\left(u_{1}\right)=u_{1}^{\prime}$ such that $y\left(u_{1}\right)=L$. Suppose that for some $i<k$ we have already placed $u_{1}, u_{2}, \ldots u_{i}$, so that $L \leq y\left(u_{1}\right) \leq$ $y\left(u_{2}\right) \leq \ldots \leq y\left(u_{i}\right)$ and there is no vertex to the West of $u_{i}$. Next we determine the place of $u_{i+1}$.

If $N\left(u_{i+1}\right) \in B_{1}^{2}$, then let $r \in \mathcal{R}$ be the ray starting at $N\left(u_{i+1}\right)$ and pointing to the Northwest. If $N\left(u_{i+1}\right) \in B_{1}^{3} \cup B_{\mathbf{x}}$, let $r \in \mathcal{R}$ be the ray starting at $N\left(u_{i+1}\right)$ and pointing to the North. In both cases, place $u_{i+1}$ on $r$ : if $u_{i}$ lies on the lefthand side of $r$, then put $u_{i+1}$ to the Northeast of $u_{i}$; otherwise, put $u_{i+1}$ to the West of $u_{i}$.

If $N\left(u_{i+1}\right) \in B_{2}$, then let $r \in \mathcal{R}$ be the ray starting at $N\left(u_{i+1}\right)$ and pointing to the North, or, if we have already placed a point on this ray, let $r$ be the other ray from $N\left(u_{i+1}\right)$, pointing to the Northwest, and proceed as before.

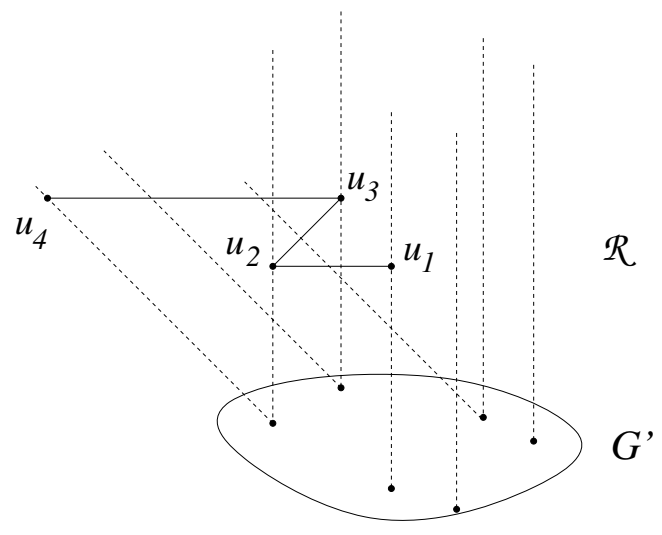

Fig. 2. Recursively place $u_{1}, u_{2}, \ldots u_{k}$ on the rays belonging to $\mathcal{R}$

SteP 4: Suppose we have already placed $u_{k}$. It remains to find the right position for $u_{0}:=v$, which has only two neighbors, $u_{1}$ and $u_{k}$. Let $r$ be the ray at $u_{1}$, pointing to the North. If $u_{k}$ lies on the left-hand side of $r$, then put $u_{0}$ on $r$ to the Northeast of $u_{k}$; otherwise, put $u_{0}$ on $r$, to the West of $u_{k}$.

During the whole procedure, we have never placed a vertex on any edge, and all other conditions of Theorem 2 are satisfied

Remark that the $y$-coordinates of the vertices $u_{0}=v, u_{1}, \ldots, u_{k}$ are at least $L$ higher than the $y$-coordinates of all vertices in $G \backslash C$. If we fix $G, C$, and $\mathbf{x}$, 

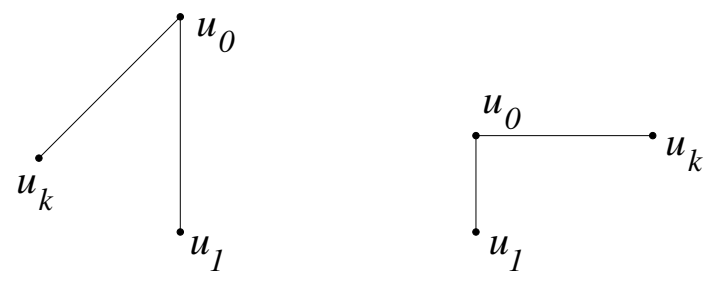

Fig. 3. Find the right position for $u_{0}$

and let $L$ tend to infinity, the coordinates of the vertices given by the above Embedding Procedure $(G, C, \mathbf{x}, L)$ change continuously.

\section{Proof of Theorem 1}

We are going to show that any graph $G$ with maximum degree three permits a straight-line drawing using only the four basic directions (of slopes $0, \pi / 2, \pi / 4$, and $-\pi / 4)$, and perhaps one further direction, which is almost vertical and is used for at most one edge in each connected component of $G$.

Denote the connected components of $G$ by $G_{1}, G_{2}, \ldots, G_{t}$. If a component $G_{s}$ is not three-regular, or if it is a complete graph with four vertices, then, by Theorem 2 , it can be drawn using only the four basic directions. If $G_{s}$ has a $\Theta$-subgraph, one can argue in the same way as in Case 2 of the proof of Theorem 2: Embed recursively the rest of the graph, and attach to it a small copy of this subgraph such that all edges of the $\Theta$-subgraph, as well as the edges used for the attachment, are parallel to one of the four basic directions. Actually, in this case, $G_{s}$ itself can be drawn using the four basic directions, so the fifth direction is not needed.

Thus, in the rest of the proof we can assume that $G_{s}$ is three-regular, it has more than four vertices, and it contains no $\Theta$-subgraph. For simplicity, we drop the subscript and we write $G$ instead of $G_{s}$. Choose a shortest cycle $C=$ $u_{0} u_{1} \ldots u_{k}$ in $G$. Each vertex of $C$ has precisely one neighbor in $G \backslash C$. On the other hand, as in the proof of the last case of Theorem 2, all vertices in $G \backslash C$ have at most two neighbors in $C$.

We distinguish two cases.

Case 1. $G \backslash C$ is a cycle. Since $G$ is three-regular, $C$ and $G \backslash C$ are of the same size and the remaining edges of $G$ form a matching between the vertices of $C$ and the vertices of $G \backslash C$. For any $i, 0 \leq i \leq k$, let $u_{i}^{\prime}$ denote the vertex of $G \backslash C$ which is connected to $u_{i}$. Denote the vertices of $G \backslash C$ by $v_{0}, v_{1}, \ldots, v_{k}$, in cyclic order, so that $v_{1}=u_{1}^{\prime}$. Then we have $v_{i}=u_{0}^{\prime}$, for some $i>1$. Apply Lemma 2.2 to $G \backslash C$ with a rationally independent assignment $\mathrm{x}$ of $x$-coordinates to the vertices $v_{1}, \ldots, v_{k}$, such that $x\left(v_{1}\right)=1, x\left(v_{i}\right)=\sqrt{2}$, and the $x$-coordinates of the other vertices are all greater than $\sqrt{2}$. (Recall that $v_{0}$ is an exceptional vertex with no assigned $x$-coordinate.) It is not hard to see that if we follow the construction described in the proof of Lemma 2.2, we also have $x\left(v_{0}\right)>\sqrt{2}$. 
Case 2. $G \backslash C$ is not a cycle. Let $u_{0}^{\prime}$ denote the neighbor of $u_{0}$ in $G \backslash C$. Since $G$ has no $\Theta$-subgraph, $u_{0}^{\prime}$ cannot be joined to both $u_{1}$ and $u_{k}$. Assume without loss of generality that $u_{0}^{\prime}$ is not connected to $u_{1}$. Let $u_{1}^{\prime}$ denote the neighbor of $u_{1}$ in $G \backslash C$.

Fix a rationally independent assignment $\mathbf{x}$ of $x$-coordinates to the vertices of degree at most two in $G \backslash C$, such that $x\left(u_{0}^{\prime}\right)=\sqrt{2}, x\left(u_{1}^{\prime}\right)=1$, and the $x$-coordinates of the other vertices are all greater than $\sqrt{2}$. Consider a drawing of $G \backslash C$, meeting the requirements of Theorem 2 .

Now in both cases, let $G^{\prime}$ denote the graph obtained from $G$ after the removal of the edge $u_{0} u_{0}^{\prime}$. Clearly $G \backslash C=G^{\prime} \backslash C$, and for any $L$, EmbedDing Procedure $\left(G^{\prime}, C, \mathbf{x}, L\right)$ gives a drawing of $G^{\prime}$. It follows from the construction, that $x\left(u_{0}\right)=x\left(u_{1}\right)=x\left(u_{1}^{\prime}\right)=1, x\left(u_{0}^{\prime}\right)=\sqrt{2}$. Therefore, for any sufficiently small $\varepsilon>0$ there is an $L>0$ such that Embedding $\operatorname{Procedure}\left(G^{\prime}, C, \mathbf{x}, L\right)$ gives a drawing of $G^{\prime}$, in which the slope of the line connecting $u_{0}$ and $u_{0}^{\prime}$ is $\frac{\pi}{2}+\varepsilon$.

We want to add the segment $u_{0} u_{0}^{\prime}$ to this drawing. Since there is no vertex with $x$-coordinate between 1 and $\sqrt{2}$, the segment $u_{0} u_{0}^{\prime}$ cannot pass through any vertex of $G$.

Summarizing: if $\varepsilon$ is sufficiently small (that is, if $L$ is sufficiently large), then each component of the graph has a proper drawing in which all edges are of one of the four basic directions, with the exception of at most one edge whose slope is $\frac{\pi}{2}+\varepsilon$. If we choose an $\varepsilon>0$ that works for all components, then the whole graph can be drawn using only at most five directions. This concludes the proof of Theorem 1.

\section{Algorithm and Concluding Remarks}

Based on the proof, it is not hard to design an algorithm to find a proper drawing, in quadratic time.

First, if our graph is a circle, we have no problem drawing it in $O(n)$ steps. If our graph has a vertex of degree one then the procedure of Case 1 of the proof of Theorem 2 requires at most $O(m)$ time when we reinsert $v_{1}, \ldots, v_{m}$.

We can check if our graph has any $\Theta$-subgraph in $O(n)$ time. If we find one, we can proceed by induction as in Case 2 of the proof of Theorem 2. We can reinsert the $\Theta$-subgraph as described in Case 2 in $O(1)$ time.

Now assume that we have a vertex $v$ of degree two. Execute a breadth first search from any vertex, and take a minimal vertex of degree two, that is, a vertex $v$ of degree two, all of whose descendants are of degree three. If there is an edge in the graph connecting a descendant of $v$ with a non-descendant, then there is a cycle through $v$; we can find a minimal one with a breadth first search from it and proceed as in Case 4. Otherwise, $v$ can play the role of $w$ in Case 3, and we can proceed recursively.

Finally, if the graph is 3-regular, then we draw each component separately, except the last step, when we have to pick an $\epsilon$ small enough simultaneously for 
all components, this takes $O(n)$ steps. We only have to find the greatest slope and pick an $\varepsilon$ such that $\frac{\pi}{2}+\varepsilon$ is even steeper.

We believe that this algorithm is far from being optimal. It may perform a breadth first search for each induction step, which is probably not necessary. One may be able to replace this step by repeatedly updating the results of the first search. We cannot even rule out that the problem can be solved in linear time.

\section{References}

1. J. Barát, J. Matoušek, and D. Wood: Bounded-degree graphs have arbitrarily large geometric thickness, Electronic J. Combinatorics 13/1 (2006), R3.

2. G. Di Battista, P. Eades, R. Tamassia, and I.G. Tollis: Graph Drawing, Prentice Hall, Upper Saddle River, N.J., 1999.

3. V. Dujmović, M. Suderman, and D.R. Wood: Really straight graph drawings, in: Graph Drawing (GD'04), J. Pach, ed., Lecture Notes in Computer Science 3383, Springer-Verlag, Berlin, 2005, 122-132.

4. C. A. Duncan, D. Eppstein, and S.G. Kobourov: The geometric thickness of low degree graphs, in: Proc. 20th ACM Symp. on Computational Geometry (SoCG'04), ACM Press, 2004, 340-346.

5. M. Engelstein: Drawing graphs with few slopes, Research paper submitted to the Intel Competition for high school students, New York, October 2005.

6. D. Eppstein: Separating thickness from geometric thickness, in: Towards a Theory of Geometric Graphs (J. Pach, ed.), Contemporary Mathematics 342, Amer. Math. Soc, Providence, 2004, 75-86.

7. J. Pach and D. Pálvölgyi: Bounded-degree graphs can have arbitrarily large slope numbers, Electronic J. Combinatorics 13/1 (2006), N1.

8. P. Ungar: On diagrams representing maps, J. London Math. Soc. 28 (1953), 336342.

9. G. A. Wade and J. H. Chu: Drawability of complete graphs using a minimal slope set, The Computer J. 37 (1994), 139-142. 